\title{
Surgical Management of Osteoblastoma of the Spine: Case Series and Review of the Literature
}

\author{
Benjamin D. ELDER, C. Rory GOODWIN, Thomas A. KOSZTOWSKI, Sheng-Fu L. LO, Ali BYDON, \\ Jean-Paul WOLINSKY, George I. JALLO, Ziya L. GOKASLAN, Timothy F. WITHAM, Daniel M. SCIUBBA
}

The Johns Hopkins University School of Medicine, Department of Neurosurgery, Baltimore, Maryland, USA

\section{ABSTRACT}

AIM: To describe the demographics, clinical and radiologic presentation, surgical considerations, and clinical outcomes associated with spinal osteoblastoma.

MATERIAL and METHODS: A spinal tumor database of 522 patients treated surgically at a single institution between January 2002 and June 2012 was analyzed and five patients with spinal osteoblastoma were identified and included in this study. Basic demographic and epidemiological data were recorded. Tumor characteristics, surgical parameters, and clinical follow-up data were noted.

RESULTS: The mean follow-up was 21.9 months. There were four males and one female, and the mean age at diagnosis was 28.4 years. There was a mean reported symptom length of 26.4 months prior to diagnosis. There were three cervical lesions, one lumbar lesion, and one sacrococcygeal lesion. One patient was Enneking stage III and four patients were Enneking stage II. Based on spinal instability neoplastic score criteria, two patients were stable and three patients were potentially unstable. Four patients had intralesional gross total resections and one patient had an en bloc marginal resection. All patients remained neurologically stable or improved postoperatively. A single patient had recurrence of a previously resected osteoid osteoma with progression to osteoblastoma.

CONCLUSION: Aggressive surgical resection of spinal osteoblastoma is suggested to minimize the risk of tumor recurrence, although this may still occur even with en bloc resection. Patients rarely present with preoperative spinal instability, but surgical fusion is often required due to removal of structural elements of the spine during resection. Of note, osteoid osteoma may progress to osteoblastoma in the spine despite prior resection.

KEYWORDS: Osteoblastoma, En bloc resection, Enneking stage

\section{INTRODUCTION}

Osteoblastoma is a rare primary tumor, accounting for around $1 \%$ of all bone tumors and up to $3 \%$ of benign bone tumors (13). They are located in the vertebral column in up to $40 \%$ of patients (18).

Surgical resection is the mainstay treatment for spinal osteoblastoma, with high recurrence rates with subtotal resection (12). Some have advocated intralesional gross total resections for Enneking stage II tumors and en bloc resections for Enneking stage III or "aggressive" variants $(2,12)$. As osteoblastomas typically involve the posterior elements of the spine (15), achieving a gross total resection or en bloc resection often involves removal of the facets or pedicles. The Spine Oncology Study Group (SOSG) recently developed the spinal instability neoplastic score (SINS) as a method to identify preoperative spinal instability in spinal oncology, though it has primarily been applied to metastatic disease (10). Although spinal insta- 
bility is rarely encountered preoperatively, as is the case with many primary tumors of the spine, following excision of structural elements of the spine, spinal fusion may be required.

In this report, we present a retrospective review of five patients with surgically treated spinal osteoblastoma in order to describe the demographics, clinical and radiologic presentation, surgical considerations, and clinical outcomes associated these tumors. Additionally, a SINS analysis was undertaken to assess the degree of preoperative instability in these tumors. Additionally, we describe in detail the cases of an osteoid osteoma progressing to osteoblastoma and an osteoblastoma with a secondary aneurysmal bone cyst, which are two rarely reported events in the literature.

\section{MATERIAL and METHODS}

This study was approved by the Institutional Review Board at our center. 522 patients were operated on for primary or metastatic spinal tumors at our center between January 2002 and June 2012. Five patients underwent surgical treatment for osteoblastoma of the spine and were included in this study. The demographic information, preoperative characteristics, surgical treatment, and outcomes were retrospectively reviewed for each patient following protocols approved by the Institutional Review Board. Survival data was obtained from the Social Security Death Master File, accessioned online on $9 / 11 / 2014$.

All patients included in the study presented with histologically confirmed osteoblastoma of the spine. Two patients were diagnosed with a computed tomography (CT)-guided biopsy, and five patients were diagnosed with open surgical resection. One patient with osteoid osteoma developed a recurrence and was diagnosed with osteoblastoma at the time of repeat resection.

For each patient, basic demographic and epidemiological data including age, gender, duration of symptoms preoperatively, pain at diagnosis, neurologic deficit at diagnosis, NSAID use prior to diagnosis, and scoliosis at time of diagnosis were recorded. Additionally, tumor characteristics including pathology, spinal location, Enneking stage (benign), WeinsteinBoriani-Biagini (WBB) staging (3), SINS (10), and tumor volume were obtained. Tumor volume was calculated using the approximated formula of an ellipsoid mass volume, $[1 / 2 x$ height $x$ width $x$ depth]. Surgical characteristics were also recorded and included preoperative embolization, surgical approach, type of resection (intralesional versus en bloc), whether a fusion was performed, surgical duration, estimated blood loss (EBL), postoperative transfusion, length of hospital stay, postoperative complications and postoperative neurologic function. The EBL and transfusion information could not be located for one patient and the data was eliminated from the reported results for these parameters. Finally, follow-up data included duration of follow-up, tumor recurrence, neurologic function at last follow-up, mortality, and status at last followup.

\section{RESULTS}

There were five patients who were diagnosed with osteoblastoma. Table I displays the demographic information and preoperative characteristics of each patient. There were four males and one female diagnosed with osteoblastoma, and the mean age at diagnosis was 28.4 years (range 15-57 years).

All patients presented with regional pain at the time of diagnosis, but only $40 \%$ of the patients were using NSAIDs at the time of diagnosis. No patients presented with scoliosis. There was a mean reported symptom length of 26.4 months (range 6-60 months) prior to diagnosis. A single patient presented with a neurologic deficit preoperatively. This patient had a large sacrococcygeal osteoblastoma and presented with bowel, bladder, and sexual dysfunction, as well as left plantar flexion weakness (3/5 strength) (Figure 1A,B).

The tumor characteristics for each patient are found in Table II. There were three cervical lesions, one lumbar lesion, and one sacrococcygeal lesion. The cervical lesions all involved a single level, the lumbar lesion involved two levels, and the sacral lesion was multilevel. One patient was Enneking stage III and four patients were Enneking stage II. Based on SINS criteria, two patients were characterized as stable and three patients were characterized as potentially unstable. The mean tumor volume was $57.8 \mathrm{cc}$ (range 2.2-265.5 cc).

Table I: Demographics and Preoperative Characteristics of Patients

\begin{tabular}{cccccccc}
\hline Patient \# & $\begin{array}{c}\text { Age at } \\
\text { Diagnosis } \\
\text { (yrs) }\end{array}$ & Gender & $\begin{array}{c}\text { Length of Symptoms } \\
\text { Preoperatively } \\
\text { (months) }\end{array}$ & $\begin{array}{c}\text { Pain at } \\
\text { Diagnosis }\end{array}$ & $\begin{array}{c}\text { Use of } \\
\text { NSAIDs }\end{array}$ & $\begin{array}{c}\text { Scoliosis at } \\
\text { Diagnosis }\end{array}$ & $\begin{array}{c}\text { Neurologic } \\
\text { Deficit at } \\
\text { Diagnosis }\end{array}$ \\
\hline $\mathbf{1}$ & 57 & M & 24 & Yes & No & No & Yes $^{*}$ \\
\hline $\mathbf{2}$ & 15 & F & 6 & Yes & No & No & No \\
\hline $\mathbf{3}$ & 27 & M & 36 & Yes & No & No & No \\
\hline $\mathbf{4}$ & 27 & M & 60 & Yes & Yes & No & No \\
\hline $\mathbf{5}$ & 16 & M & 6 & Yes & Yes & No & No \\
\hline
\end{tabular}

*Neurologic deficit included bowel, bladder, and sexual dysfunction, and left plantar flexion weakness (3/5 strength).

Abbreviations: \#: number; yrs: years; NSAIDs: non-steroidal anti-inflammatory drugs, M: Male, F: Female. 
Table II also lists the surgical approach and fusion characteristics. A posterior approach to the spine was used in all patients. A single patient had a preoperative embolization performed. Four patients had intralesional gross total resections and one patient had an en bloc marginal resection (sacrectomy) with sacrifice of the bilateral S2-5 nerve roots (Figure 1C). All patients had a posterior fusion though Patient 2 had an in situ non-instrumented fusion. For patient 1, an en bloc marginal resection was planned given concern for osteosarcoma and due to the apparent vascularity of the mass. The tumor appeared to involve S2 through the coccyx on imaging, and it was initially felt that an instrumented fusion would not be necessary. However, following osteotomies below the S1 nerve roots, it was apparent that the tumor had invaded the S1 vertebral body, requiring a more proximal cut between L5 and S1. Given that the S1-coccyx segments had been completely removed, it was felt that the patient would require lumbosacropelvic reconstruction with an instrumented fusion.
All patients remained neurologically stable or improved postoperatively. The mean estimate blood loss (EBL) was $2038 \mathrm{cc}$ (range 300-6000 cc), and two patients required blood transfusions during the hospitalization. The mean hospital length of stay was 6.4 days (range 2-19 days).

No patients received postoperative chemotherapy or radiation. A single patient in the osteoblastoma series (20\%) developed a postoperative complication. The patient with the large sacrococcygeal osteoblastoma developed a wound dehiscence requiring surgical revision on postoperative day 11 .

The mean length of follow-up was 21.9 months. The patient with the large sacrococcygeal osteoblastoma developed a local recurrence 43 months postoperatively, with a $25.2 \mathrm{cc}$ lesion noted at L5-S1 on the left (Figure 1D, E). A CT-guided biopsy was performed due to concern for osteosarcoma, but osteoblastoma was confirmed on the biopsy. A preoperative embolization was performed, followed by a repeat intralesional gross total resection with removal of the medial aspect of
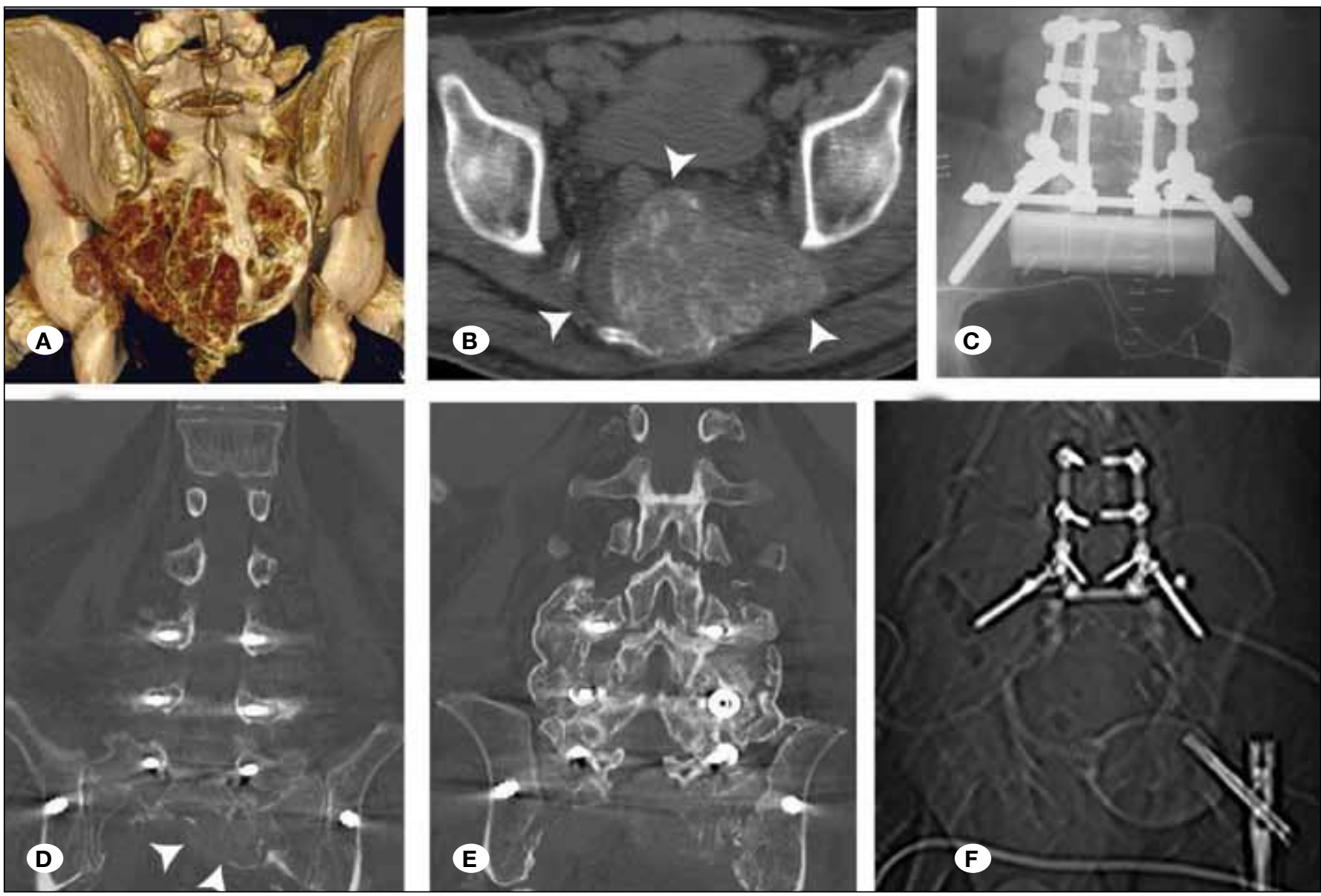

Figure 1: Representative images for patient 1. Preoperative A) 3-D CT reconstruction and B) axial CT demonstrating large sacrococcygeal lytic, expansile lesion with cortical destruction, internal calcifications, and associated soft tissue component, depicted by the arrowheads. An en bloc marginal resection with a sacrectomy was performed and reconstruction with L3-pelvis instrumentation and a transiliac strut allograft was performed (C). A recurrent lesion was noted 43 months later, with involvement of the remaining sacrum and at L5, depicted by the arrowheads (D). A solid posterolateral fusion was noted (E). Following a preoperative embolization, an intralesional gross total resection was performed and the medial aspect of the transiliac strut allograft was removed (F). Pathology confirmed recurrent osteoblastoma. 


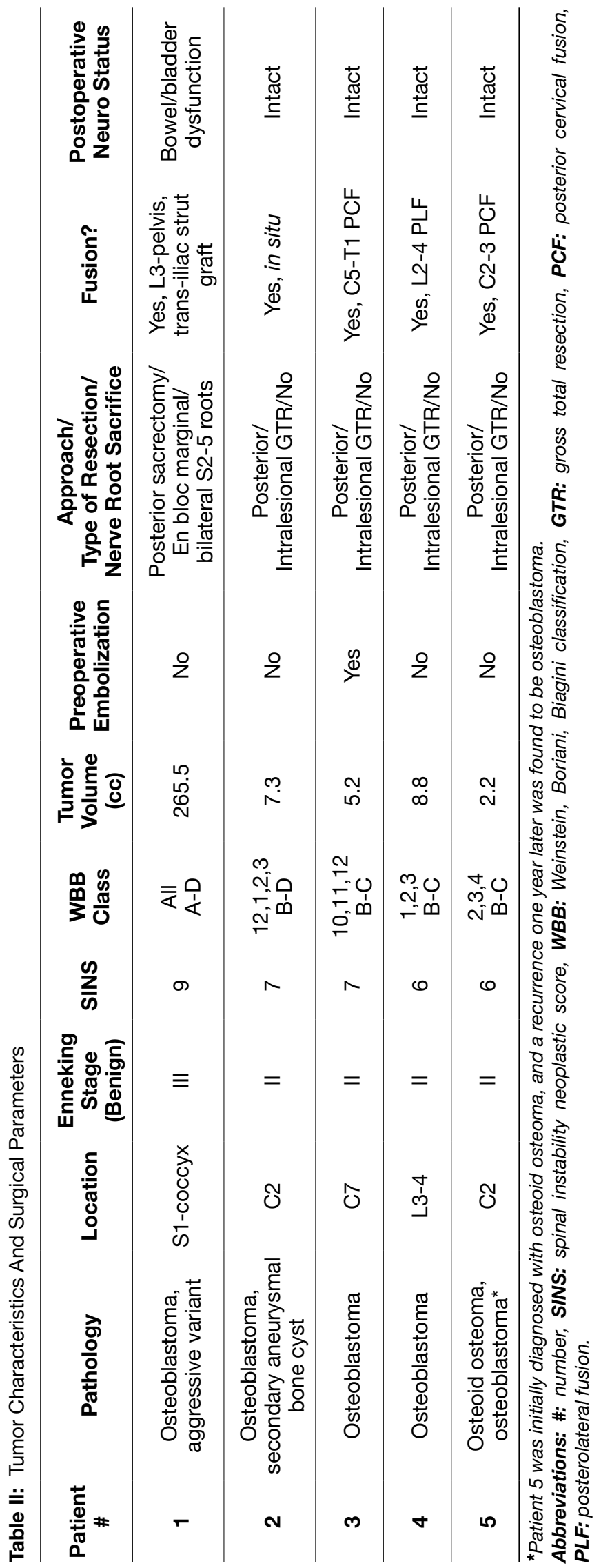

the transiliac strut allograft (Figure 1F). He ultimately expired 5 months after the recurrence, but the cause of death was not known. Other than the single patient death, the remaining four patients were alive with no systemic evidence of local or systemic disease at last follow-up.

Patient 2 presented with neck pain and suspected to have an aneurysmal bone cyst (Figures $2 \mathrm{~A}-\mathrm{C}$ ). A resection of the lamina and left lateral mass was performed (Figure 2D) and pathology demonstrated osteoblastoma with secondary aneurysmal bone cyst. Patient 5 was found to have a lesion in the left C2 lamina and lateral mass (Figure 3A, B), pathology demonstrated osteoid osteoma at the initial resection, at which time a C2-3 fusion was also performed (Figure 3C, D). However, he developed a recurrence 13 months postoperatively (Figure $3 E-G)$, and a repeat resection was performed (Figure $3 \mathrm{H}$ ). The recurrent lesion had transitioned to an osteoblastoma, confirmed by pathology. The original pathology was reanalyzed and still found to be consistent with osteoid osteoma suggesting progression to osteoblastoma.

\section{DISCUSSION}

Although several other larger series of spinal osteoblastomas have been reported $(2,8,16,17,20,21,24)$, to the best of our knowledge, this is the first primary tumor series to assess preoperative spinal stability using the SINS criteria. Additionally, this series includes the first case to demonstrate pathology-confirmed progression of osteoid osteoma to osteoblastoma in the spine. Finally, an osteoblastoma with a secondary aneurysmal bone cyst is reported, which has been rarely described in the spinal clinical literature previously.

As described above, this manuscript presents the first case of pathology-confirmed progression of osteoid osteoma to osteoblastoma in the spine. Osteoid osteoma and osteoblastoma were originally felt to be variations, predominantly of size, of the same tumor type. However, current clinical consensus is that these are two separate entities with different clinical and radiologic characteristics as well as different risks of progression $(14,15)$. Two cases of progression of spinal osteoid osteoma to osteoblastoma were previously reported, though no pathology-confirmed diagnosis of osteoid osteoma was obtained. For instance, Bruneau et al. (4) reported the case of a 25-year-old patient with an $8 \mathrm{~mm}$ lesion in the left $\mathrm{C} 1$ lateral mass, concerning for osteoid osteoma. The patient initially refused surgery, but seven years later, he had increased pain and the lesion had grown to $16 \mathrm{~mm}$. Surgical resection was performed with pathology demonstrating osteoblastoma. A similar case was reported in a 16-year-old patient in which a 6 $\mathrm{mm}$ lesion was identified in the right $\mathrm{C} 6$ pedicle concerning for osteoid osteoma. The patient originally refused surgery and one year later the lesion had increased in size and was found to be osteoblastoma following surgical resection (5). As such, there is controversy as to whether these "progressions" truly represent pathologic change, or if the original smaller lesions were simply incorrectly assumed to be osteoid osteomas without pathology confirmation $(6,19)$. The results reported here are interesting as biopsy samples were obtained at each resection and reviewed by the same senior musculoskeletal 
pathologist. Additionally, after the recurrent specimen was felt to be osteoblastoma, the original specimen was re-reviewed and still felt to be osteoid osteoma. However, this has previously been reported in the appendicular skeleton, as Bettelli et al. reported the case of a 24-year-old patient diagnosed with a femoral osteoid osteoma based on pathology, and later diagnosed with osteoblastoma following surgical resection for a recurrence 8 months later (1).
Patient 2 in this series was found to have an osteoblastoma of $\mathrm{C} 2$ with a secondary aneurysmal bone cyst, which has been rarely reported in the literature. Although a retrospective review of 55 patients, with osteoblastoma in any region of the skeleton, reported a $14.5 \%$ rate of secondary aneurysmal bone cysts (7), there have otherwise only been anecdotal reports in the literature, with rare reports of this dual pathology in the spine. For instance, Tarantino et al. (25) presented a
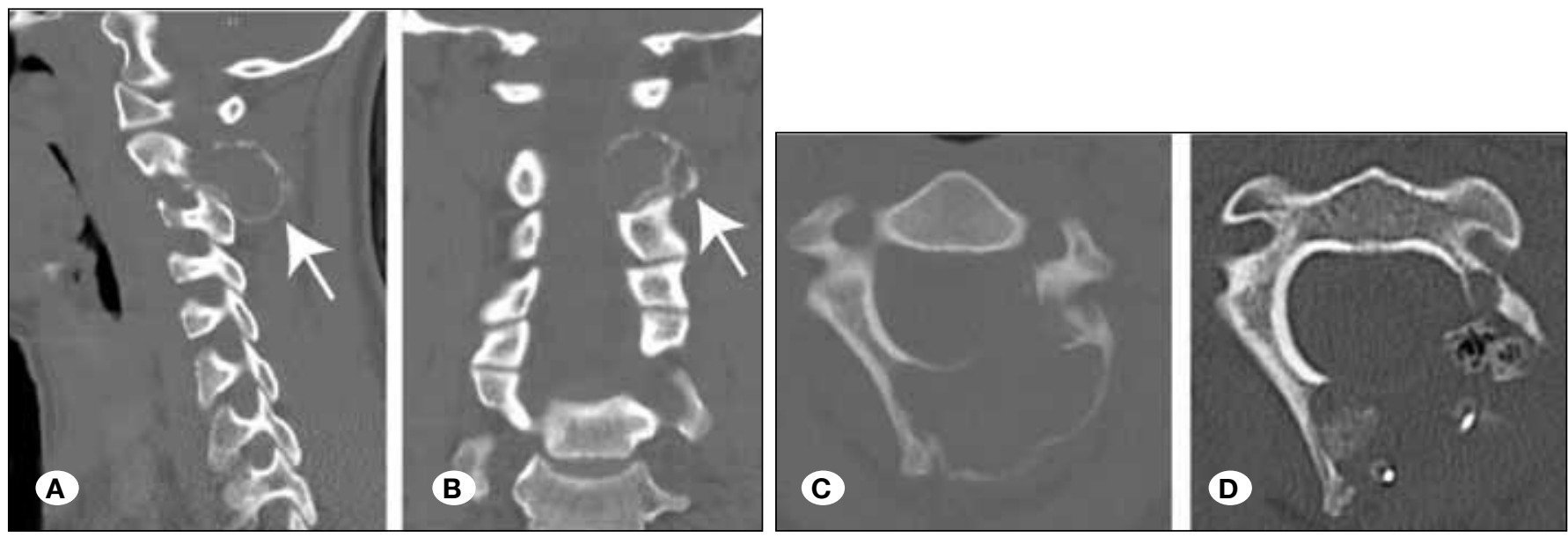

Figure 2: Representative images for patient 2. Preoperative A) sagittal, B) coronal, and C) axial CT images demonstrate C2 lesion (arrows) in left lamina and lateral mass suspicious for aneurysmal bone cyst with an expansile osteolytic appearance with thin sclerotic margins. D) Resection of the lamina and lateral mass with curettage was undertaken and an in situ non-instrumented was performed.
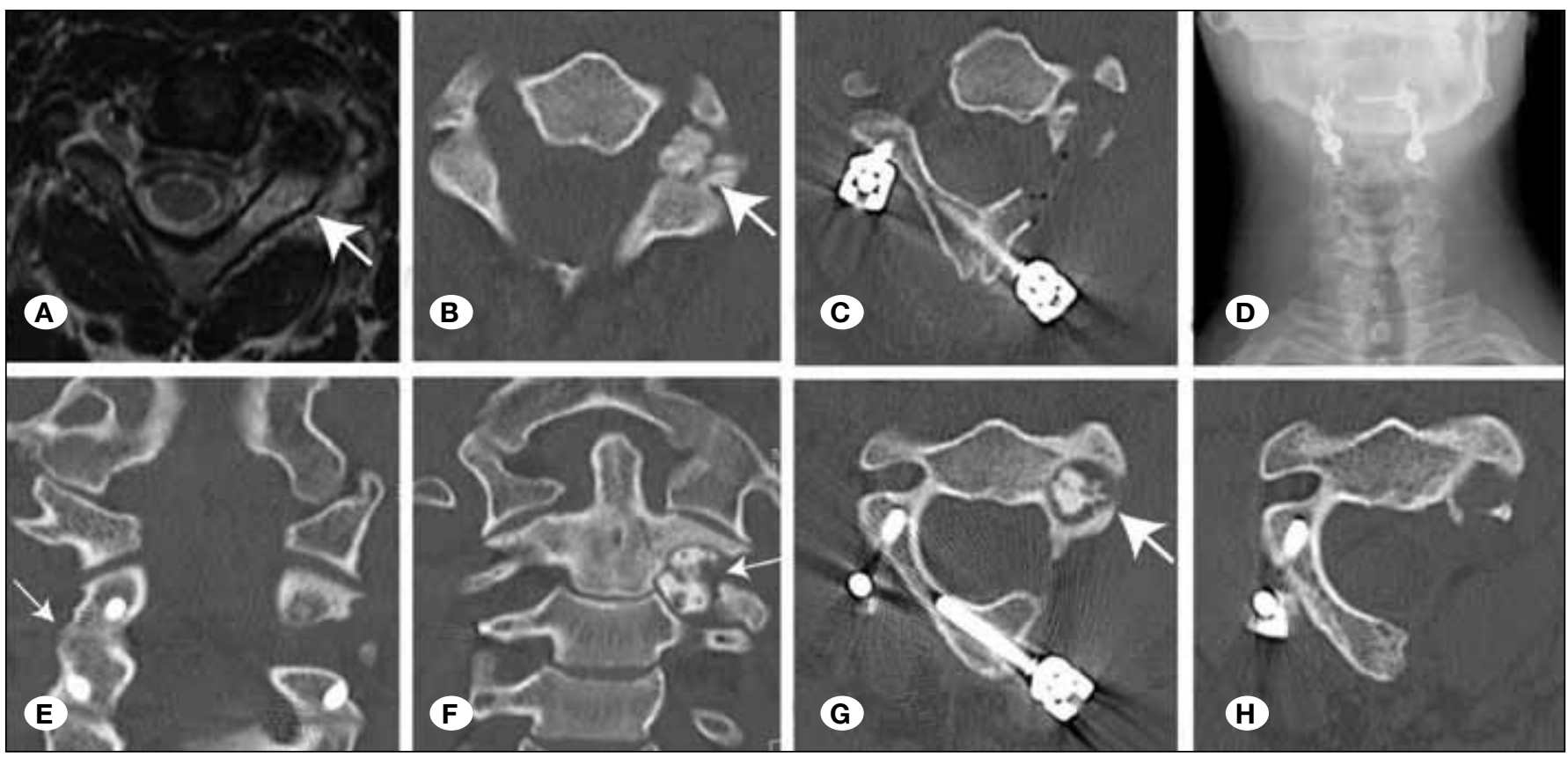

Figure 3: Representative images for patient 3. Preoperative axial A) T2-weighted MRI and B) CT scan images demonstrate hypointense lesion extending to the left pedicle and lateral mass of C2 with hyperintense signal in the lamina (A), and a well-circumscribed lesion noted on CT (B), as depicted by the arrows. An intralesional gross total resection was performed with removal of the lamina, lateral mass, and pedicle on the left and skeletonization of the vertebral artery. A C2-3 posterior fusion was performed with a translaminar C2 screw on the left (C-D). A recurrent lesion was noted 13 months later surrounding the vertebral artery (F-G), depicted by the arrows, and solid fusion was noted on the right side (E). A repeat resection was carried out with further skeletonization of the left vertebral artery and removal of the $\mathrm{C} 2-3$ instrumentation on the left $\mathbf{( H )}$. 
15-year-old patient with an osteoblastoma of the odontoid process with a secondary aneurysmal bone cyst, while Ramme and Smucker reported a 19-year-old patient with an osteoblastoma and secondary aneurysmal bone cyst in the posterior elements of C2 (22). Additionally, osteoblastoma with secondary aneurysmal bone cyst has been reported in the sacrum (26) and the posterior cranial fossa (11).

Histologically, they are composed of osteoid and woven bone, and frequently contain highly vascular regions (7) and numerous osteoblasts. They most commonly affect the thoracolumbar spine $(15,23)$, although frequent involvement of the cervical spine has also been reported (20). They have a greater incidence in males and are most commonly diagnosed in the second decade of life, with a prolonged duration of symptoms prior to presentation of up to an average of 20 months $(12,15)$. Patients typically present with localized pain in the neck or back, the pain may increase nocturnally. It typically improves with aspirin or non-steroidal antiinflammatory drugs (NSAIDs).

The majority of the demographic and clinical results of our series were not substantially different than those of prior reported series $(2,8,16,17,20,21,24)$. According to a recent review (15), there is typically a male predominance, and this was observed in our series at a rate of $4: 1$. Additionally, the mean duration of symptoms prior to diagnosis was described as approximately 20 months (15), similar to the 26 months observed in this series, with $40 \%$ of the patients controlling their pain with NSAIDs. Additionally, the rate of neurologic deficit at presentation has ranged between $25-70 \%$ (15), with a $20 \%$ rate in this series, though only observed in the sacral lesion. In a series of 18 patients with sacral osteoblastomas, 4 patients $(22 \%)$ were found to have neurologic deficits.

However, the mean age of diagnosis, at 28.4 years, with a patient in the sixth decade of life, was substantially older than has been reported in previous series, with the most common age of presentation in the second decade (15). Only one other series identified an older patient (60 years) with an average of 23 years of age (2), and most patients ranged between 15-30 years of age. The older age of the patients as well as cervical predominance in our series may explain why no patients presented with a deformity. Rates of scoliosis typically range between $4.5-100 \%$ at diagnosis, according to a recent review (15), though this is typically an issue more with thoracolumbar lesions in juvenile patients.

An area of controversy following resection of spinal osteoblastomas is whether spinal fusion should be performed following tumor resection. For instance, there is concern for postlaminectomy kyphosis especially after resection of C2 lesions in the posterior elements. However, the precise definition of spinal instability has been unclear in the clinical literature. The SOSG defined spinal instability as "a loss of spinal integrity as a result of a neoplastic process that is associated with movement-related pain, symptomatic or progressive deformity, and/or neural compromise under physiologic loads (10). The SOSG recently developed the SINS as a method to identify preoperative spinal instability in spinal oncology, though it has primarily been applied to metastatic disease. Based on SINS criteria, three patients were identified as potentially unstable and two patients were identified as stable, and this was the first time in the literature that a SINS analysis was applied to a primary spinal tumor. Although spinal instability is rarely encountered preoperatively, as is the case with many primary tumors of the spine, following excision of structural elements of the spine, spinal fusion may be required. The resections of the cervical and lumbar lesions in this series all involved removal of the facet joints or pedicles to allow for gross total resection. As such, all patients in the series were fused, although one patient had a non-instrumented fusion. The pediatric patients require further scrutiny, as they are at greater risk of postlaminectomy kyphosis (9), but there may be reluctance to limit the mobility of these younger patients with spinal fusion. Therefore, great care should be taken during surgical resection to minimize disruption of the facet joints and attempt to reattach the paraspinal muscles to the posterior elements, if fusion is not pursued.

The type of surgical treatment has also been debated and correlated with surgical grading $(2,12,27)$. In our series, the patient with the aggressive variant, Enneking stage III, was treated with a marginal en bloc resection, while the remaining Enneking stage II patients were treated with intralesional gross total resections. According to a recent systematic review by Harrop et al. (12), the recurrence rates for intralesional subtotal resections or biopsies, marginal gross total resections, or en bloc resections were $93 \%, 15 \%$, and $20 \%$ respectively. Additionally, the "aggressive" or Enneking stage III lesions have been reported to recur in up to $50 \%$ with subtotal resections. Therefore, they recommended intralesional gross total resection for Enneking stage II lesions, and en bloc resections for Enneking stage III lesions when "anatomically feasible," and this was the treatment strategy pursued in our series. Yin et al. (27) also identified tumor size $>3 \mathrm{~cm}$ as predictive of increased risk of tumor recurrence on multivariate analysis. It is also important to at least obtain a biopsy of any recurrence as sarcomatous degeneration to osteosarcoma has been described (12).

With a recurrent lesion, as observed in the sacral mass in this series, radiation was not pursued, as it has not been shown to decrease the risk of recurrence following subtotal resection (12). Surgical resection remains the mainstay treatment. However, radiation can potentially be reserved as a treatment option for Enneking stage III tumors if there is no feasible surgical option (2). Similarly, a limited, if any, role for chemotherapy has been identified in the treatment of osteoblastoma.

A major limitation of this study is the retrospective nature with a small number of patients, as well as a single cohort with lack of a control group. However, even in this small series, rarely reported events in the literature including progression of osteoid osteoma to osteoblastoma and an osteoblastoma with a secondary aneurysmal bone cyst merit reporting.

\section{- CONCLUSION}

Aggressive surgical resection should be considered for osteoblastoma to minimize the risk of tumor recurrence, although 
this may still occur despite en bloc resection. Patients rarely present with preoperative spinal instability, but surgical fusion is often required due to removal of structural elements of the spine during resection. Of note, osteoid osteoma may progress to osteoblastoma in the spine despite prior resection.

\section{SOURCES of FINANCIAL SUPPORT and INDUSTRY AFFILIATIONS}

Benjamin D. ELDER: This article reflects the views of the author and should not be construed to represent the FDA's views or policies. C. Rory GOODWIN: Supported by the UNCF-Merck Postdoctoral Fellowship and an Award from the Burroughs Wellcome Fund. Ali BYDON: Recipient of a research grant from DePuy Spine and serves on the clinical advisory board of Medlmmune, LLC Ziya L. GOKASLAN: Disclosures of stock ownership in US Spine and Spinal Kinetics, consulting, speaking and teaching for the $A O$ Foundation and research support from DePuy, NREF, AO Spine, and AO North America. Timothy F. WITHAM: Recipient of research funding from Eli Lily and Company; research support from the Gordon and Marilyn Macklin Foundation. Daniel M. SCIUBBA: Consultant for DePuy, Medtronic, Nuvasive, and Globus.

\section{REFERENCES}

1. Bettelli G, Tigani D, Picci P: Recurring osteoblastoma initially presenting as a typical osteoid osteoma. Report of two cases. Skeletal Radiol 20: 1-4, 1991

2. Boriani S, Amendola L, Bandiera S, Simoes CE, Alberghini $M$, Di Fiore $M$, Gasbarrini A: Staging and treatment of osteoblastoma in the mobile spine: A review of 51 cases. Eur Spine J 21: 2003-2010, 2012

3. Boriani S, Weinstein JN, Biagini R: Primary bone tumors of the spine. Terminology and surgical staging. Spine 22:1036-1044, 1997

4. Bruneau M, Polivka M, Cornelius JF, George B: Progression of an osteoid osteoma to an osteoblastoma. Case report. J Neurosurg Spine 3: 238-241, 2005

5. Cappuccio M, De lure F, Amendola L, Corghi A, Gasbarrini A: Cervical osteoid osteoma progression to osteoblastoma. Spine J 14: 1070-1071, 2014

6. Chotel F, Franck F, Solla F, Dijoud F, Kohler R, Berard J, Abelin Genevois K; French Sarcoma Group - Bone Tumour Study Group: Osteoid osteoma transformation into osteoblastoma: Fact or fiction? Orthop Traumatol Surg Res 98(6 Suppl):S98-104, 2012

7. Della Rocca C, Huvos AG: Osteoblastoma: Varied histological presentations with a benign clinical course. An analysis of 55 cases. Am J Surg Pathol 20: 841-850, 1996

8. Denaro V, Denaro L, Papalia R, Marinozzi A, Di Martino A: Surgical management of cervical spine osteoblastomas. Clin Orthop Relat Res 455:190-195, 2007

9. Deutsch H, Haid RW, Rodts GE, Mummaneni PV: Postlaminectomy cervical deformity. Neurosurg Focus 15: E5, 2003

10. Fisher CG, DiPaola CP, Ryken TC, Bilsky MH, Shaffrey Cl, Berven SH, Harrop JS, Fehlings MG, Boriani S, Chou D, Schmidt MH, Polly DW, Biagini R, Burch S, Dekutoski MB, Ganju A, Gerszten PC, Gokaslan ZL, Groff MW, Liebsch NJ, Mendel E, Okuno SH, Patel S, Rhines LD, Rose PS, Sciubba
DM, Sundaresan N, Tomita K, Varga PP, Vialle LR, Vrionis FD, Yamada Y, Fourney DR: A novel classification system for spinal instability in neoplastic disease: An evidence-based approach and expert consensus from the Spine Oncology Study Group. Spine 35: E1221-1229, 2010

11. Han X, Dong Y, Sun K, Lu Y: A huge occipital osteoblastoma accompanied with aneurysmal bone cyst in the posterior cranial fossa. Clin Neurol Neurosurg 110: 282-285, 2008

12. Harrop JS, Schmidt MH, Boriani S, Shaffrey Cl: Aggressive "benign" primary spine neoplasms: Osteoblastoma, aneurysmal bone cyst, and giant cell tumor. Spine 34: S3947, 2009

13. Healey JH, Ghelman B: Osteoid osteoma and osteoblastoma. Current concepts and recent advances. Clin Orthop Relat Res 204:76-85, 1986

14. Jackson RP, Reckling FW, Mants FA: Osteoid osteoma and osteoblastoma. Similar histologic lesions with different natural histories. Clin Orthop Relat Res 128:303-313, 1977

15. Kan P, Schmidt MH: Osteoid osteoma and osteoblastoma of the spine. Neurosurg Clin North Am 19:65-70, 2008

16. Kaner T, Sasani M, Oktenoglu T, Aydin S, Ozer AF: Osteoid osteoma and osteoblastoma of the cervical spine: The cause of unusual persistent neck pain. Pain Phys 13: 549-554, 2010

17. Li H, Zou X, Xue Q, Egund N, Lind M, Bunger C: Anterior lumbar interbody fusion with carbon fiber cage loaded with bioceramics and platelet-rich plasma. An experimental study on pigs. Eur Spine J 13: 354-358, 2004

18. Marsh BW, Bonfiglio M, Brady LP, Enneking WF: Benign osteoblastoma: Range of manifestations. J Bone Joint Surg (Am) 57: 1-9, 1975

19. Morton KS, Quenville NF, Beauchamp CP: Aggressive osteoblastoma. A case previously reported as a recurrent osteoid osteoma. J Bone Joint Surg (Br) 71:428-431, 1989

20. Nemoto O, Moser RP, Jr., Van Dam BE, Aoki J, Gilkey FW: Osteoblastoma of the spine. A review of 75 cases. Spine 15: 1272-1280, 1990

21. Ozaki T, Liljenqvist U, Hillmann A, Halm H, Lindner N, Gosheger G, Winkelmann W: Osteoid osteoma and osteoblastoma of the spine: Experiences with 22 patients. Clin Orthop Relat Res 397:394-402, 2002

22. Ramme AJ, Smucker JD: Balancing spinal stability and future mobility in the cervical spine: Surgical treatment of a case of osteoblastoma with secondary aneurysmal bone cyst. Spine J 11: e5-12, 2011

23. Ropper AE, Cahill KS, Hanna JW, McCarthy EF, Gokaslan ZL, Chi $\mathrm{JH}$ : Primary vertebral tumors: A review of epidemiologic, histological, and imaging findings, Part I: Benign tumors. Neurosurgery 69: 1171-1180, 2011

24. Ruggieri P, Huch K, Mavrogenis AF, Merlino B, Angelini A: Osteoblastoma of the sacrum: Report of 18 cases and analysis of the literature. Spine 39: E97-E103, 2014

25. Tarantino R, Piccirilli M, Anichini G, Delfini R: Benign osteoblastoma of the odontoid process of the axis with secondary aneurysmal bone cyst component: A case report. Neurosurg Rev 31: 111-115, 2008

26. Vade A, Wilbur A, Pudlowski R, Ghosh L: Case report 566: Osteoblastoma of sacrum with secondary aneurysmal bone cyst. Skeletal Radiol 18: 475-480, 1989

27. Yin $\mathrm{H}$, Zhou W, Yu H, Li B, Zhang D, Wu Z, Liu T, Xiao J: Clinical characteristics and treatment options for two types of osteoblastoma in the mobile spine: A retrospective study of 32 cases and outcomes. Eur Spine J 23:411-416, 2014 